Discrimination à l'embauche des jeunes en Ile-de-France : Un diplôme plus élevé compense-t-il une origine maghrébine?

EMILIA ENE JONES

TEPP - Travail, Emploi et Politiques Publiques - FR CNRS 3126 


\title{
Discrimination à l'embauche des jeunes en Ile-de-France : Un diplôme plus élevé compense-t-il une origine maghrébine?
}

\author{
Emilia Ene ${ }^{1}$
}

Décembre 2010

\section{Résumé}

A l'aide d'un testing nous évaluons l'ampleur de la discrimination à l'embauche envers les jeunes d'origine étrangère (maghrébine) en Ile-de-France. Nous avons mesuré l'effet de l'origine (française ou maghrébine) croisé avec la variable «diplôme » sur les chances d'accéder aux entretiens d'embauche en réponse à des offres d'emploi pour le poste de technicien de maintenance.

Afin de réaliser cette étude, nous avons construit 3 profils de demandeurs d'emploi : deux jeunes hommes d'origine maghrébine et un jeune homme d'origine française. Nous avons délibérément renforcé les caractéristiques productives d'un des deux candidats d'origine maghrébine par rapport aux autres candidats. Ainsi, l'un de ces deux candidats d'origine maghrébine affiche un niveau de formation plus élevé que les autres candidats. Il détient un diplôme de niveau BTS alors que les autres détiennent un diplôme de niveau BAC.

Nous avons envoyé 441 candidatures en réponse à 147 offres d'emploi en Île-de-France sur la période fin juillet 2010 - fin août 2010.

Les résultats obtenus mettent en évidence des pratiques discriminatoires envers les candidats maghrébins. Ces pratiques ne sont que faiblement compensées par une formation plus élevée car le candidat d'origine maghrébine plus qualifié a un moindre accès aux entretiens d'embauche que le candidat français mais plus de chances que l'autre candidat d'origine maghrébine. Alors que le diplôme augmente les chances d'accéder aux entretiens d'embauche, l'origine maghrébine reste pourtant pénalisante.

Mots clés : discrimination à l'embauche, testing, origine ethnique, qualification, diplôme.

Classification JEL : C81, C93, J15, J71.

\footnotetext{
${ }^{1}$ Université de Paris-Est, Erudite, CEE et TEPP (FR CNRS n³126), 5 Boulevard Descartes, Bâtiment Bois de l'Étang - Bureau C239, Champs-sur-Marne, 77454 Marne-la-Vallée Cedex 2; Centre d'Etudes de l'Emploi Groupe de recherche Celeste, bureau 212, 29 Promenade Michel Simon 93166 Noisy-le Grand cedex. E-mail : emillia.ene@gmail.com, tél : +33145926819.

Ce travail est issu d'un mémoire de master 2 de l'Université de Paris Est Marne la Vallée réalisé sous la direction de Yannick L'Horty et Pascale Petit, que je tiens à remercier.
} 


\section{Introduction}

La discrimination envers certains groupes de la population est un problème majeur pour notre société et elle fait l'objet de nombreuses études (Riach et Rich, 2002 ; Kenney et Wissoker, 1994 ; Bertrand et Mullainathan, 2004 ; Cediey et Foroni, 2007 ; Duguet E. et all. 2007, 2010, par exemple). L'exercice d'évaluation de la discrimination reste compliqué à cause d'éventuelles caractéristiques inobservables ou mal identifiées. Afin d'éviter l'utilisation abusive de ce concept, une définition précise de la notion de discrimination s'impose.

James Heckman (1998) parle d'une situation discriminatoire lorsque deux travailleurs pourvus de caractéristiques productives parfaitement identiques et qui se différencient juste par des caractéristiques non productives, ne bénéficient pas de mêmes attributs (accès à l'emploi, à la formation, promotions, niveau des salaires, etc.) de la part d'une entreprise.

Sur le marché du travail, on parle de discrimination à l'embauche lorsque des chercheurs d'emploi sont traités de manière différente sur une base de critères de sélection qui manquent de justification objective et raisonnable à l'égard du poste à pourvoir.

Les travaux sur le problème théorique posé par la discrimination partent d'une même définition de la discrimination comme traitement différencié des travailleurs avec des productivités identiques. Elles peuvent être classées en deux grandes catégories. Les théories fondées sur des préférences discriminatoires, il s'agit d'une aversion des employeurs, des salariés ou des consommateurs pour les minorités (Becker, 1957). Les théories fondées sur l'idée que la discrimination est une conséquence du manque d'information des employeurs sur la productivité des employés lors de l'embauche (Arrow, 1972 et 1973, McCall, 1972 et Phelps, 1972). Pour Phelps (1972), l'appréciation de la productivité repose sur des signaux individuels. Il parle de discrimination statistique : comme conséquence de l'existence d'une composante inobservée de la productivité des candidats, l'employeur fonde l'appréciation des compétences inobservables sur une évaluation de l'individu lors de l'embauche (réussite aux tests d'embauche, diplômes...) et sur ses croyances sur la productivité moyenne du groupe démographique auquel appartient le candidat. Pour Arrow (1973), les employeurs ont des croyances fondées sur l'observation ou encore des préjugés relatifs à la corrélation entre le sexe et la performance. 
Toutes ces différentes études théoriques montrent que l'identification et l'évaluation de la discrimination sont difficiles. Une méthode bien adaptée s'impose donc pour évaluer de manière satisfaisante la discrimination à l'embauche.

Les données disponibles habituellement (l'enquête Emploi ou l'Enquête sur la structure des salaires de l'Insee, par exemple) ne sont pas suffisantes car l'évaluation de la discrimination est un exercice complexe qui suppose des comparaisons «toutes choses étant égales par ailleurs ». Or les données issues de sources statistiques existantes posent la difficulté méthodologique de l'inadéquation des données d'enquête ou administratives (caractéristiques des candidats aux postes, déclarations non fiables des employeurs et des candidats, dans la réalité 2 candidatures à un même emploi ne sont jamais identiques). En effet, si certaines caractéristiques des individus sont observables (par exemple le sexe ou l'âge) pour d'autres ce n'est pas le cas (comme par exemple la motivation des individus).

Un exemple d'étude qui utilise des données statistiques publiques est celui de R. Aeberhardt, D. Fougère, J. Pouget et R. Rathelot (2010). En utilisant des données de l'Enquête Emploi 2005-2008, ils montrent des inégalités sur le marché du travail des enfants d'immigrés provenant des pays du Maghreb et d'Europe du Sud : «Les Français ayant au moins un parent immigré originaire du Maghreb ont des taux d'emploi inférieurs de 18 points et des salaires $13 \%$ inférieurs à ceux de français dont les deux parents sont français de naissance ». Quant à la comparaison des groupes d'origine d'Europe du Sud, ces inégalités sont plus faibles (écart d'accès à l'emploi de 0.7 et écart de salaire de $2 \%$ ).

Nous remarquons que, lorsque l'on cherche à évaluer la discrimination en utilisant des données d'enquêtes ou administratives, nous observons seulement le résultat du processus de recrutement (comme, par exemple, la proportion des membres d'un certain groupe démographique, ici les enfants d'immigrés provenant des pays du Maghreb et d'Europe du Sud). Mais nous ne pouvons pas distinguer de manière précise si ce résultat est la conséquence d'une différence des caractéristiques productives des candidats, d'une discrimination à l'embauche ou peut-être d'une autocensure des candidats à l'embauche.

Afin de contrôler complètement l'hétérogénéité inobservée et de pouvoir réaliser des comparaisons «toutes choses égales par ailleurs » une approche purement expérimentale est nécessaire. Comme solution à ces difficultés, la méthode du testing est une méthode adéquate qui permet de collecter des données à l'aide d'une expérience contrôlée ${ }^{2}$. Il s'agit de

\footnotetext{
${ }^{2}$ Pour une étude plus détaillée sur la procédure du testing voir les publications de Petit (« Comment évaluer la discrimination à l'embauche ? » 2003), Heckman (1998) et Aeberhardt, Pouget, Fougere, Rathelot (« Discrimination à l'embauche, quels apports des procédures de testing ? »2009).
} 
construire des candidatures ( $\mathrm{CV}$ et lettres de motivation) équivalentes en tous points à l'exception de la variable à tester. Les candidatures ainsi construites sont envoyées aux mêmes offres d'emploi. Le taux de réponse des candidats est l'instrument utilisé pour comparer l'accès aux entretiens d'embauche des différents candidats. Pour comparer l'accès à l'emploi des candidats, si les candidatures sont retenues par les employeurs, les responsables de l'étude peuvent choisir d'envoyer de faux candidats aux entretiens d'embauche.

Cette méthode comporte pourtant des limites. Les plus importantes sont les suivantes. Les données recueillies sont des données expérimentales. "Elles indiquent de façon fiable l'ampleur de la discrimination à l'embauche à un moment donné du temps, dans le champ couvert par l'expérience mais elles ne peuvent en aucun cas fournir un indicateur de l'état des discriminations sur l'ensemble du marché du travail »(De Schutter, 2001 ; Duguet et al, 2007). Ensuite, comme le souligne Heckman (1998) il est difficile d'isoler la discrimination fondée sur l'aversion de la discrimination statistique. Les résultats obtenus ne peuvent pas être généralisés sur l'ensemble du marché du travail et leur interprétation doit être prudente. Enfin, dans une étude plus récente, Neumark (2010) montre que, si les correspondance-testing incluent explicitement des variations dans la qualité des candidatures, certains résultats peuvent être mal interprétés comme preuves de discrimination.

La méthode du testing est utilisée pour des travaux sur la discrimination à l'embauche en raison de l'origine ethnique depuis une vingtaine d'années.

Riach et Rich (1991), en Australie, comparent l'accès à l'emploi entre autochtones et minorités grecques et vietnamiennes pour des postes à faible qualification (vendeurs, secrétaires). Leurs résultats indiquent une discrimination à l'embauche significative dont les grecs et les vietnamiens ont été victimes. Cependant, la discrimination apparaît inférieure pour les grecs $(8,8 \%)$ que pour les vietnamiens (27.4\%). Aux États-Unis, Kenney et Wissoker (1994) réalisent simultanément à Chicago et à San Diego un test pour comparer l'accès aux entretiens d'embauche et à l'emploi des jeunes hommes d'origine hispanique et anglo-saxonne. Les résultats obtenus montrent que la discrimination se situe surtout au niveau de l'accès aux entretiens : alors qu'ils trouvent une discrimination significative envers les candidats hispaniques dans l'accès aux entretiens d'embauche, cette discrimination diminue lors du passage d'un entretien pour l'obtention d'un emploi.

Des travaux plus récents traitent l'effet cumulé de l'origine et d'autres variables sur les chances d'accès à l'emploi. 
Bertrand et Mullainathan (2004), aux États-Unis, effectuent un «correspondance testing » en utilisant des candidats blancs et noirs d'origine anglo-saxonne ou afro-américaine pour des emplois à faible qualification (caissier, guichetier à la poste, vendeur). L'origine ethnique des candidats est signalée sur la candidature par le prénom à forte consonance anglo-saxonne ou afro-américaine. Ils trouvent une importante discrimination à l'encontre des candidats noirs (taux de succès des candidats portant un prénom et un nom à consonance afro-américaine de $6.45 \%$ contre $9.65 \%$ pour des candidats portant un prénom et un nom à consonance anglosaxonne). De plus, ils montrent que seuls les blancs sont avantagés par une candidature de qualité plus élevée.

En France, Cediey et Foroni (2007) ont effectué à Lille, Lyon, Marseille, Nantes, Paris et Strasbourg une étude pour le Bureau International du Travail (BIT), « Les discriminations en raison de "l'origine" dans les embauches en France : une enquête nationale par tests de discrimination selon la méthode du BIT ». Les tests de discrimination ont été réalisés sur la période fin 2005 - mi 2006 en utilisant les types d'emplois de basses et moyennes-basses qualifications dans les domaines de l'hôtellerie-restauration, de la vente et du commerce, des services aux entreprises ou aux collectivités, des services à la personne, des transports, de l'accueil et du secrétariat, du bâtiment et des travaux publics, de la santé et de l'action sociale. L'origine ethnique des candidats est signalée sur la candidature par le prénom et le nom à forte consonance hexagonale ancienne, maghrébine ou noire africaine.

Les résultats montrent une nette discrimination à l'encontre des candidats d'origine maghrébine et noire africaine.

Duguet E., N. Léandri, Y. L'Horty, P. Petit (2007) réalisent un test d'accès aux entretiens d'embauche en mesurant de façon simultanée les effets de l'origine (française ou marocaine) suggérée par la consonance du nom de famille et du prénom, de la nationalité (française ou marocaine) et du lieu de résidence. Les auteurs se sont intéressés à des postes de serveurs et de comptables. Leurs résultats montrent que dans les deux cas, afficher sur la candidature un nom et un prénom à consonance maghrébine est plus pénalisant qu'afficher une nationalité maghrébine. De plus, ils montrent une discrimination différenciée à l'encontre des maghrébins selon les deux postes. En effet, les auteurs montrent que pour un poste de serveur, les chances d'obtenir un entretien d'embauche sont au moins 3 fois plus fortes pour les candidats d'origine française que pour les candidats d'origine ou de nationalité maghrébine. Pour les postes de comptables, ces différences apparaissent plus fortes (« Les candidats de nationalité et d'origine maghrébines doivent en moyenne envoyer dix fois plus de curriculum 
vitae pour obtenir autant d'invitations à des entretiens d'embauche que les candidats dont les noms et prénoms évoquent l'origine française »).

Plus récemment, Duguet E., Y. L’Horty, L. du Parquet, P. Petit, F. Sari (2011) évaluent les effets croisés de l'origine (française, maghrébine, africaine subsaharienne et asiatique) et du sexe sur les chances d'accéder aux entretiens d'embauche. Ils se sont intéressés à des postes qualifiées et en tension des informaticiens de niveau BAC+5. Ils montrent que, les français d'origine étrangère ont une probabilité plus faible d'accéder aux entretiens d'embauche quel que soit leur sexe.

Une autre étude intéressante est celle de A. M Ahmed, L. Andersson et M. Hammarstedt (2009). Ils indiquent que la discrimination ethnique semble exister dans le marché des transferts de petites entreprises (self-employment), donc aussi en dehors du marché du travail conventionnel. En utilisant deux acheteurs fictifs potentiels, dont un avec un nom typiquement suédois et un avec un nom typiquement maghrébin, ils ont répondu sur internet aux annonces de transferts de petites entreprises en Suède. Ils ont constaté que les vendeurs discriminent les acheteurs avec un nom maghrébin. Ainsi, les acheteurs avec un nom maghrébin ont obtenu moins de réponses positives que les acheteurs avec un nom suédois.

L'objectif de notre étude est de mettre en évidence l'existence éventuelle et l'ampleur de la discrimination à l'embauche en raison de l'origine tout en tenant compte d'un niveau de qualification (diplôme) volontairement différent entre certains candidats. Comme dans l'expérience de Bertrand et Mullainathan (2004), nous nous intéressons aussi à augmenter la qualité des candidatures mais juste pour un certain candidat. Ainsi, pour ce candidat, nous avons accordé une formation supérieure (un diplôme de plus) car conformément à la théorie du capital humain les chances d'accès à l'embauche augmentent avec le niveau de formation. En comparaison, Bertrand et Mullainathan ont accordé des compétences en langues étrangères, des honneurs ou une certaine expérience militaire, des écoles d'été ou des expériences de travail pendant l'école, des expériences de bénévolat, des compétences supplémentaires en informatique.

\section{Apports de notre étude}

Cette étude a comme objectif l'évaluation des résultats d'un test d'accès aux entretiens d'embauche des jeunes hommes d'origine française et maghrébine afin de mettre en évidence l'existence éventuelle et l'ampleur des pratiques discriminatoires envers des personnes d'origine étrangère (maghrébine). 
Tout d'abord, nous innovons par le fait que nous avons volontairement augmenté les caractéristiques productives d'un des candidats par rapport aux autres candidats en faisant varier la variable «qualification ». Puisque les travaux de référence ont démontré l'existence de pratiques discriminatoires à l'encontre des personnes d'origine maghrébine, nous sommes sortis du champ « toutes choses étant égales par ailleurs » et nous avons délibérément renforcé les caractéristiques productives d'un des deux candidats d'origine maghrébine par rapport aux autres candidats en lui accordant un diplôme de plus.

Ainsi, un premier candidat est d'origine maghrébine signalée par la consonance maghrébine de son nom et de son prénom. Il affiche sur sa candidature une formation plus élevée que les autres candidats : un diplôme de niveau BTS. Un deuxième candidat est d'origine maghrébine, ce qui est signalée par la consonance maghrébine de son nom et de son prénom et il affiche sur sa candidature un diplôme du niveau BAC. Un troisième candidat est d'origine française signalée par la consonance française de son nom et de son prénom et il affiche sur sa candidature un diplôme du niveau BAC.

Toutes les autres caractéristiques des candidats sont similaires. Ainsi, les derniers deux candidats se différencient entre eux juste par la caractéristique non productive « origine ethnique ». Le premier candidat se différencie des autres candidats aussi par une caractéristique productive, une formation plus élevée, menée à renforcer ses chances d'accéder à l'emploi.

Dans ce contexte, nous cherchons à répondre aux questions suivantes : dans le cas où un français d'origine maghrébine est mieux formé qu'un français autochtone, quel est l'effet sur ses chances d'accès à l'embauche? Est-ce qu'on va trouver une discrimination à l'embauche envers le candidat d'origine maghrébine et si oui, quelle est son ampleur?

Ensuite, nous innovons par le fait que nous avons cherché à voir si une discrimination existe dans l'ordre des réponses fait aux candidats. Cette approche est à notre connaissance une approche originale non encore exploitée.

Les trois types de candidatures ont donc été construits pour être envoyés en réponse aux mêmes offres d'emplois, dans les mêmes entreprises. Nous avons envoyé 441 réponses à 147 offres d'emploi sur la période fin juillet - fin août 2010 en utilisant le site Pôle Emploi.

Les types d'emploi utilisés dans le cadre de notre étude sont des emplois qualifiés (BTS, BAC PRO) dans le secteur d'activité de la maintenance, le métier de technicien de maintenance.

En tenant compte des limites du testing, nous mentionnons que les résultats obtenus sur ce secteur d'activité ne peuvent pas être considérés comme un indicateur de l'ampleur de la discrimination sur l'ensemble du marché du travail. 


\section{Nature de l'expérience}

Dans notre étude, nous avons testé la variable de l'origine des candidats signalée par la consonance française ou maghrébine du nom et du prénom des candidats (cf. tableau 1). Cette variable est testée en tenant compte du renforcement des chances d'accéder à l'emploi pour un des deux candidats d'origine maghrébine: il bénéficie d'un diplôme BTS en plus par rapport aux deux autres candidats.

Toutes les autres caractéristiques sont similaires, sans être totalement identiques pour limiter le risque de détection par les recruteurs.

Tableau 1

Origine ethnique de candidats

\begin{tabular}{|l|lll|}
\hline Candidature & Nationalité & Origine & Consonance nom et prénom \\
\hline $1(\mathrm{FMM})$ & Française & Maghrébine & Consonance maghrébine \\
\hline $2(\mathrm{FMM})$ & Française & Maghrébine & Consonance maghrébine \\
\hline $3(\mathrm{FFF})$ & Française & Française & Consonance française \\
\hline
\end{tabular}

Lecture : Les deux premiers candidats (FMM) ont la nationalité française, les noms et prénoms à consonance maghrébine. Le troisième candidat $(F F F)$ a la nationalité française, ses nom et prénom à consonance française.

\section{Construction des candidatures écrites}

Nous avons choisi de tester l'effet de l'origine maghrébine sur les chances d'accès aux entretiens d'embauche pour le métier de technicien de maintenance en électrotechnique. Conformément à l'Enquête Emploi 2010, dans ce secteur il y a 3.29\% des employés d'origine maghrébine, juste après les employés d'origine de l'Europe du Sud qui sont en proportion de $5.46 \%$. Dans des proportions plus faibles, nous trouvons des employés d'origine d'Europe du Nord (1.13\%) et de l'Est (1.40\%) et, en dessous de 1\%, des employés d'origine du ProcheOrient, de Laos, Vietnam et Cambodge, du reste de l'Afrique et du reste du monde. La proportion des employés diplômés BTS est de $15.31 \%$ tandis que les employés diplômés Bac Pro sont en proportion de $14.44 \%$.

Le métier de technicien de maintenance est un métier pour lequel on note des difficultés de recrutement. Conformément à plusieurs bulletins mensuels d'information sur le marché du travail en Ile de France $\left(n^{\circ} 26\right.$, août $2006 ; n^{\circ} 37$, juillet - août $2007 ; n^{\circ} 43$, août $2008 ; n^{\circ} 45$, février 2009) les recrutements jugés difficiles se situaient particulièrement dans le domaine du bâtiment, de la cuisine et de la santé. La part des recrutements de techniciens du bâtiment envisagée jugée difficile est montée de 59,4\% à 75,2\% sur la période 2006 - 2008. 
La grande demande de travailleurs pour un poste de technicien de maintenance et les difficultés de recrutement le transforment en métier en tension. De plus, la spécialisation dans l'électrotechnique nous permet d'accéder à une palette large de postes : électrotechnicien (code ROME I1304-I1309); électromécanicien (code ROME I1304-I1309); technicien / agent d'entretien et de maintenance du bâtiment (code ROME I1203); technicien d'essais en électronique (H1504); technicien d'installations de matériels électroniques (code ROME I1305); technicien de maintenance industrielle (code ROME I1304), etc. La qualification et les prérequis correspondants à ces codes de métiers correspondent aux qualifications et aux expériences professionnelles accordées aux candidats.

Un tel métier doit normalement limiter les pratiques discriminatoires et les nombres de refus des employeurs. Pour ces raisons, nous avons choisi d'utiliser le métier de technicien de maintenance en électrotechnique dans le cadre de notre étude.

Nous avons choisi de tester l'origine maghrébine parce que, selon plusieurs études relatives à ce sujet, les plus grandes difficultés d'accéder à l'emploi sont subies par les personnes originaires de ces pays et par les enfants de ces personnes (Richard J. L., 2006 ; Silberman R.et Fournier I., 2006, Duguet et al, 2007). Ainsi, tandis que la variable « origine ethnique » (dans notre cas l'origine maghrébine) est une caractéristique non productive elle génère pourtant des pratiques discriminatoires. Mais que va-t-il se passer quand une caractéristique productive « entre en jeu » pour renforcer les chances d'un candidat d'origine maghrébine?

Afin de répondre à cette question, nous avons choisi d'adapter notre approche et faire varier la variable «formation professionnelle» entre les candidats (cf. tableau 2). Ainsi, nous avons construit trois candidatures qui permettent de former trois couples de candidats.

Tableau 2

\section{Qualification de candidats}

\begin{tabular}{|lll|}
\hline Candidature & Poste & Qualification \\
\hline $1($ FMM $)$ & $\begin{array}{l}\text { Technicien de } \\
\text { maintenance }\end{array}$ & $\begin{array}{l}\text { BTS électrotechnique } \\
\text { Bac Pro électrotechnique, énergie, équipements } \\
\text { communicants BEP métiers de l'électrotechnique }\end{array}$ \\
\hline $2($ FMM) & $\begin{array}{l}\text { Technicien de } \\
\text { maintenance }\end{array}$ & $\begin{array}{l}\text { Bac Pro électrotechnique, énergie, équipements } \\
\text { communicants BEP métiers de l'électrotechnique }\end{array}$ \\
\hline $3($ FFF $)$ & $\begin{array}{l}\text { Technicien de } \\
\text { maintenance }\end{array}$ & $\begin{array}{l}\text { Bac Pro électrotechnique, énergie, équipements } \\
\text { communicants BEP métiers de l'électrotechnique }\end{array}$ \\
\hline
\end{tabular}


Dans le premier couple (2FMM, 3FFF), les candidats se distinguent par l'origine ethnique signalée sur la candidature par la consonance de leurs noms et prénoms. Les deux candidats ont une nationalité française mais l'un d'entre eux affiche un nom et un prénom qui suggèrent une origine maghrébine. Les nom et prénom affichés par l'autre candidat suggèrent une origine française. Les deux candidats ont un CV de qualité équivalente et affichent donc des caractéristiques productives similaires (mêmes qualifications BAC).

Similairement au premier couple, les candidats du deuxième couple (1FMM, 3FFF), se différencient par l'origine ethnique (française et maghrébine) et, particulièrement pour le candidat d'origine maghrébine, par sa formation professionnelle supérieure. Ainsi, le candidat d'origine française affiche sur sa candidature une formation du niveau BAC alors que le candidat d'origine maghrébine affiche une formation du niveau BTS. Toutes les autres caractéristiques sont similaires. Le dernier couple (1FMM, 2FMM) est formé des deux candidats d'origine maghrébine, l'un des deux affichant une meilleure formation que le second.

$\mathrm{Si}$, dans une telle situation, nous trouvons une préférence pour le candidat d'origine française qui est moins qualifié, cela suggérerait un niveau de très grande ampleur de discrimination envers les personnes d'origine maghrébine. Au contraire, si nous trouvons une préférence pour le candidat maghrébin qui est le plus formé, cela peut suggérer une réponse de l'employeur économiquement rationnelle à la supériorité donnée par sa formation.

En utilisant ces deux couples de candidats, nous cherchons à trouver et analyser quelles sont les chances d'accès aux entretiens d'embauche pour un candidat d'origine maghrébine par rapport à un candidat d'origine française qui détienne des caractéristiques productives équivalentes ou inférieures. Le choix des noms et des prénoms des candidats est fait en tenant compte de leurs origines ethniques et de leurs âges. Ainsi, nous avons choisi des noms et des prénoms de forte consonance maghrébine et française qui ont été beaucoup utilisés à l'époque de leur naissance (cf. tableau 3).

Tableau 3

Identité des candidats

\begin{tabular}{|lllll|}
\hline Candidature & Nom et Prénom & $\begin{array}{l}\text { Consonance du } \\
\text { Nom et Prénom }\end{array}$ & Sexe & Diplôme \\
\hline $1($ FMM $)$ & MESSAI Zahid & Maghrébine & Masculin & BTS \\
\hline $2($ FMM $)$ & BELAHDEB Nessim & Maghrébine & Masculin & BAC \\
\hline $3($ FFF $)$ & BERNARD Sylvain & Française & Masculin & BAC \\
\hline
\end{tabular}


Toutes les autres caractéristiques sont similaires, comme nous le développons par la suite.

\section{Les similitudes :}

Toutes les similitudes sont formulées différemment sur chaque CV, pour limiter le risque de détection. Par exemple l'âge est directement affiché pour un des candidats. Pour les autres deux candidats, l'âge est suggéré par la date de naissance ou par l'année de naissance sur leur candidature.

Les trois candidats sont des jeunes hommes de nationalité française, célibataires et sans enfant. Ils sont tous trois âgés de 28 ans. Ils résident tous en Île-de-France dans des zones hors ZUS similaires économiquement et socialement du code postal 93 (cf. tableau 4), pour limiter le risque que la zone de résidence ait une influence sur le choix du recruteur.

Tableau 4

\section{Lieux de résidence des candidats}

\begin{tabular}{|lll|}
\hline Candidature & Localité & Code postal \\
\hline $1(\mathrm{FMM})$ & Bondy & 93140 \\
\hline $2(\mathrm{FMM})$ & Le Blanc Mesnil & 93156 \\
\hline $3(\mathrm{FFF})$ & Noisy-le-Sec & 93130 \\
\hline
\end{tabular}

Ils détiennent tous un permis de conduire $\mathrm{B}$ et ils affichent une mobilité en Île-de-France sur leur candidature. En conformité avec leur âge, ils ont une vaste expérience professionnelle d'environ douze années. Le candidat ayant un diplôme de plus, affiche sur sa candidature une expérience professionnelle acquise en alternance pendant ces deux années d'études supplémentaires. Ainsi, l'expérience professionnelle est similaire pour les trois candidats qui ont occupé les mêmes types de postes avec des tâches équivalentes.

Les trois candidats n'affichent aucune interruption (significative) dans leur travail et ils travaillent sur des postes similaires au moment de leur candidature.

\section{Les différences :}

Les trois candidatures se différencient par des éléments de mise en page sur les supports écrits (CV et lettre de motivation) : la police, les puces et la couleur d'écriture, la taille de la police, le modèle du CV et la mise en page du contenu.

Elles se différencient aussi par leur opérateur de téléphone mobile et par leur client de messagerie internet. Ainsi, chaque candidat possède un numéro de téléphone portable et une adresse électronique sur des messageries en ligne différentes (Yahoo, Gmail, Hotmail). 
L'expérience professionnelle des candidats est acquise dans des entreprises différentes situées dans des zones différentes d'Île-de-France.

Les loisirs des candidats peuvent donner naissance à des interprétations sur le caractère des candidats et sur leur comportement éventuel. En conséquence, les candidats affichent des loisirs différents mais neutres tel que le sport d'équipe, la lecture, la musique, etc.

De plus, comme nous l'avons déjà présenté, un des trois candidats affiche un diplôme de plus que les autres.

Ainsi, les candidatures construites sont similaires, plausibles et réalistes.

\section{Un test d'accès aux entretiens d'embauche}

Notre recherche vise à évaluer l'effet d'origine (française et maghrébine) sur les chances d'obtenir un entretien d'embauche. Pour ce faire, nous avons envoyé par mail 441 candidatures écrites ( $\mathrm{CV}$ et lettres de motivation) en réponse à 147 offres d'emploi disponibles sur la période fin juillet - fin août 2010.

Nous n'avons pas envoyé des candidats aux entretiens d'embauche donc les résultats montrent seulement le taux d'accès aux entretiens d'embauche, qui est « une approximation de l'accès à l'emploi » (Duguet et al, 2007). On pourrait penser qu'un test d'accès aux entretiens d'embauches est limitant car l'accès aux entretiens ne garantit pas l'obtention d'un emploi. Cependant, l'organisation d'un entretien a un coût pour l'employeur et si celui-ci décide de convoquer un candidat, c'est qu'il a des chances d'obtenir le poste. De plus, de nombreuses études (ex : Kenney et Wissoker, 1994 ; Neumark et al. 1996 ; BIT, 2007 ; Cediey et al, 2008) montrent que la discrimination apparaît dès l'accès aux entretiens d'embauche.

Le choix de ne pas envoyer des candidats aux entretiens d'embauche nous donne l'avantage de contrôler parfaitement le déroulement de notre étude. L'apparence physique et la personnalité des candidats qui sont des éléments incontrôlables par les chercheurs, n'ont pas d'influence sur les résultats du test. De plus, ce choix simplifie la collecte de données ce qui nous donne la possibilité de constituer un échantillon plus large (Riach et Rich, 1991).

\section{Sources des offres d'emplois et envoi des candidatures}

Nous avons constitué notre échantillon en consultant le site Internet du Pôle Emploi qui centralise la plus grande partie des offres d'emploi en Ile de France. 
Nous avons répondu aux offres d'emploi quelques jours après leur parution sur le site internet. Les candidatures ont été envoyées sur la période fin juillet 2010 - fin août 2010, en réponse aux offres d'emploi correspondant au profil électrotechnique.

Puisque les candidatures étaient envoyées aux mêmes offres d'emploi, nous avons choisi de les envoyer à intervalles de 45 minutes environ à partir des adresses mail de chaque candidat, afin de limiter le risque de détection. Pour contrôler cette approche méthodologique, les candidatures ont été envoyées par rotation systématique en évitant donc d'influencer le choix des entreprises pour un candidat particulier pour que l'ordre soit différent à chaque envoi.

Pour éviter un effet potentiel de la forme ou du fond des candidatures sur les réponses des employeurs, nous avons utilisé un système de permutation régulière entre les identités des candidats et les supports écrits (CV et lettre de motivation).

Ainsi, nous avons utilisé simultanément les deux approches méthodologiques (envoi des candidatures par rotation systématique et permutations entre les identités des candidats et les supports écrits) pour construire le jeu de données le plus fiable possible.

Nous avons répondu à toutes les offres d'emploi en adéquation avec les diplômes et l'expérience des candidats qui répondaient aux critères suivants :

- Type de contrat : tout contrat (CDI, CDD, Intérim);

- Postes localisés dans toute l'Île-de-France (mobilité sur toute la région);

- Secteur d'activité : Maintenance électrotechnique.

\section{Traitement des réponses des recruteurs}

La réponse est considérée positive dans le cas où le recruteur convie le candidat à un entretien ou s'il se manifeste pour obtenir plus d'informations concernant la situation présente ou les qualifications des candidats.

La réponse est considérée comme négative dans le cas où le recruteur rejette formellement la candidature ou s'il n'y a pas de réponse de la part du recruteur. Nous avons donc choisi de traiter l'absence de réponse comme des réponses négatives pour tous les candidats. Ce choix repose sur le fait que l'étude est réalisée sur une profession en tension, les entreprises étant limitées en temps dans leur recrutement. Le fait de ne contacter aucun des candidats nous indique un désintérêt pour tous les candidats. Notons que la façon dont nous interprétons l'absence de réponse influence la grandeur des résultats au sens où la discrimination paraîtrait plus élevée si l'absence de réponse n'était pas considérée comme une réponse négative. Notre 
démarche retient donc toutes les entreprises et pas seulement celles qui ont contacté les candidats.

Pour calculer la différence nette d'accès à l'embauche entre deux candidats, nous associons une probabilité à chacun des événements. On note P1 la probabilité d'une réponse positive pour le candidat 1 et négative pour le candidat 2 et P2 la probabilité d'une réponse positive pour le candidat 2 et négative pour le candidat 1 . Le ratio entre la différence de ces deux probabilités et le nombre total d'entreprises auditées représente la différence nette (DN) d'accès à l'embauche entre les deux candidats $(\mathrm{DN}=(\mathrm{P} 1-\mathrm{P} 2) / \mathrm{N})$. Un résultat positif indique que le candidat 1 a plus des chances d'être convoqué à un entretien que le candidat 2. A l'inverse, une différence nette négative signifie que le candidat 1 a moins des chances d'être convoqué à un entretien que le candidat 2 .

\section{Résultats du test}

Parmi 147 entreprises qui ont reçu des candidatures, 28\% (41 entreprises) ont envoyé un signal favorable aux candidats en leur proposant un entretien d'embauche ou en demandant des informations supplémentaires. Le taux moyen de réussite par candidat montre pourtant des disparités entre eux (cf. tableau 5). Selon leur origine et leur niveau de formation nous avons trouvé un écart important entre les candidats.

Le candidat d'origine française, ayant une formation de niveau BAC, a reçu 35 réponses positives, contre 21 réponses positives pour le candidat d'origine maghrébine, ayant les mêmes caractéristiques productives et donc le même niveau de diplôme. Le candidat d'origine maghrébine ayant une formation supérieure de niveau $\mathrm{BAC}+2$, donc un diplôme BTS, a reçu 28 des réponses positives. Il a reçu donc plus de réponses positives par rapport à l'autre candidat d'origine maghrébine. Néanmoins, par rapport au candidat d'origine française, il a reçu moins de réponses positives.

Tableau 5

Taux de réussite

\begin{tabular}{|c|c|c|c|c|c|}
\hline \multirow{3}{*}{$\frac{\text { Candidature }}{1(\mathrm{FMM})}$} & \multirow{3}{*}{$\begin{array}{l}\text { Formation des } \\
\text { candidats }\end{array}$} & \multirow{3}{*}{\begin{tabular}{|l} 
Taux de \\
réussite (\%)
\end{tabular}} & \multirow{3}{*}{$\begin{array}{l}\text { Student } \\
5,81\end{array}$} & \multicolumn{2}{|c|}{$\begin{array}{c}\text { Intervalle de confiance de } \\
\text { niveau } 90 \%\end{array}$} \\
\hline & & & & Borne inf. & Borne sup. \\
\hline & & & & 13,79 & 24,64 \\
\hline 2 (FMM) & BAC & $14,29 * * *$ & 4,94 & 9,68 & 19,23 \\
\hline 3 (FFF) & BAC & $23,81 * * *$ & 6,85 & 18,18 & 29,70 \\
\hline
\end{tabular}

Lecture : Les statistiques de Student et les intervalles de confiance ont été calculés par la méthode du bootstrap réalisée sur 10000 tirages. $* * *$ significatif au seuil de $1 \%$ 
On remarque qu'il y a deux effets distincts de part ces résultats. Tout d'abord, la qualification BTS apporte un plus au candidat maghrébin par rapport à l'autre candidat maghrébin de niveau BAC. Cependant, le candidat français n'a besoin que de son appartenance pour combler son manque de qualification par rapport au candidat maghrébin de niveau BTS.

\section{Nombre de candidatures pour être invité à un entretien d'embauche}

Comme le montre leurs taux de réussite, le candidat d'origine française reçoit une invitation d'entretien à l'embauche pour $4 \mathrm{CV}$ envoyés en moyenne, contre $7 \mathrm{CV}$ pour un candidat d'origine maghrébine ayant les mêmes caractéristiques productives. Le candidat d'origine maghrébine ayant une formation supérieure aux autres, doit envoyer 5 candidatures pour obtenir une invitation d'entretien à l'embauche. Ainsi, alors qu'il est plus formé que le candidat d'origine française, il doit envoyer plus de candidatures pour obtenir le même résultat. Ces résultats suggèrent donc une discrimination importante sur ce marché du travail, avec des écarts différents selon l'origine et la formation.

Autrement dit, selon leur origine, des écarts importants apparaissent entre les candidats : le candidat d'origine français a en moyenne 1,75 fois plus de chances d'obtenir un entretien d'embauche que le candidat d'origine maghrébine toutes choses étant égales par ailleurs. Cet écart a tendance à se réduire mais il reste quand même présent lorsqu'on compare le candidat d'origine française et le candidat d'origine maghrébine mieux formé que le premier. Ainsi, alors qu'ils sont mieux formés, les candidats d'origine maghrébine ont toujours moins de chances (1,25 fois) d'obtenir un entretien d'embauche que les candidats d'origine française.

\section{Étude de la discrimination par couple}

Pour approfondir notre analyse des résultats, nous détaillons dans cette section une analyse par couple des trois candidats : On note « $\mathrm{A} »$ le candidat d'origine maghrébine avec un BTS, « $\mathrm{B} »$ le candidat d'origine française avec un $\mathrm{Bac}$ et $« \mathrm{C} »$ le candidat d'origine maghrébine avec un BAC.

\section{Analyse du couple AB}

\section{Le fait d'être plus qualifié ne semble pas « compenser » le fait d'être maghrébin.}

En analysant le couple formé par les candidats A et B on remarque une discrimination nette de $4,76 \%$. Cette discrimination est assez forte compte tenu du fait que le candidat le plus qualifié est le candidat maghrébin (cf. tableau 6). 
Tableau 6

Discrimination nette - Couple AB : origine et formation

\begin{tabular}{|l|l|l|l|l|l|l|}
\hline Explication & $\begin{array}{l}\text { "NON" } \\
\text { aux 2 }\end{array}$ & $\begin{array}{l}\text { "OUI" } \\
\text { aux 2 }\end{array}$ & $\begin{array}{l}\text { "NON" à A } \\
\text { "OUI" à B } \\
\mathbf{( 1 )}\end{array}$ & $\begin{array}{l}\text { "NON" à B } \\
\text { "OUI" à A } \\
\mathbf{( 2 )}\end{array}$ & $\begin{array}{l}\text { No. total } \\
\text { d'offres } \\
\mathbf{( 3 )}\end{array}$ & $\begin{array}{l}\text { Discrimination } \\
\text { nette (1)-(2) / (3) }\end{array}$ \\
\hline Total nombre & 107 & 23 & 12 & 5 & 147 & 0,0476 \\
\hline Pourcentage & 72,79 & 15,65 & 8,16 & 3,40 & 100,00 & $\begin{array}{l}4,76^{*} \\
(1,71)\end{array}$ \\
\hline
\end{tabular}

Lecture : Seuils de significativité statistique * : Significatif à 10\%; **: Significatif à 5\%. *** : Significatif à $1 \%$. Sur 147 entreprises, 107 ont répondu négativement et 23 ont répondu positivement aux 2 candidats. 12 entreprises ont répondu négativement au candidat d'origine maghrébine (A) et positivement au candidat d'origine française (B). 5 entreprises ont répondu négativement au candidat d'origine française et positivement à l'autre candidat. La discrimination nette à l'encontre du candidat maghrébin plus diplômé est donc de $4.76 \%$ [(12 - 5)/147]*100.

On note qu'un résultat égal entre les deux candidats aurait déjà été une présomption de discrimination car on s'attend à ce que le candidat ayant la meilleure formation donc la productivité potentielle la plus importante soit auditionné plus souvent. Le fait d'être plus qualifié ne semble pas «compenser » le fait d'être maghrébin pour les recruteurs.

\section{Analyse du couple BC}

L'analyse du couple BC indique une discrimination nette de 9,52\% envers le candidat d'origine maghrébine (cf. tableau 7). La discrimination est bien supérieure à celle du candidat maghrébin ayant un BTS. Rappelons tout de même que sans discrimination les résultats d'accès à l'embauche entre les deux candidats B et C auraient dû être identiques car leurs caractéristiques productives sont équivalentes.

Tableau 7

Discrimination nette - Couple BC : origine

\begin{tabular}{|l|l|l|l|l|l|l|}
\hline Explication & $\begin{array}{l}\text { "NON" } \\
\text { aux 2 }\end{array}$ & $\begin{array}{l}\text { "OUI" } \\
\text { aux 2 }\end{array}$ & $\begin{array}{l}\text { "NON" à B } \\
\text { "OUI" à C }\end{array}$ & $\begin{array}{l}\text { "NON" à C } \\
\text { "OUI" à B }\end{array}$ & Echantillon & $\begin{array}{l}\text { Discrimination } \\
\text { nette }\end{array}$ \\
\hline Total nombre & 110 & 19 & 2 & 16 & 147 & 0,0952 \\
\hline Pourcentage & 74,83 & 12,93 & 1,36 & 10,88 & 100,00 & $\begin{array}{l}9,52 * * * \\
(3,42)\end{array}$ \\
\hline
\end{tabular}

Lecture : * Significatif à 10\%; ** : Significatif à 5\%. ***: Significatif à $1 \%$. 


\section{Analyse du couple AC}

\section{La formation donne plus de chances d'accéder à un emploi}

L'analyse du dernier couple AC montre un écart net de 4,76\% (cf. tableau 8). On ne parle pas de discrimination ici car la seule caractéristique distinguant les candidats est une variable productive (la qualification). Assez logiquement, les recruteurs préfèrent le candidat d'origine maghrébine le mieux formé.

Tableau 8

Ecart net - Couple AC : formation

\begin{tabular}{|l|l|l|l|l|l|l|}
\hline Explication & $\begin{array}{l}\text { "NON" } \\
\text { aux 2 }\end{array}$ & $\begin{array}{l}\text { "OUI" } \\
\text { aux 2 }\end{array}$ & $\begin{array}{l}\text { "NON" à A } \\
\text { "OUI" à C }\end{array}$ & $\begin{array}{l}\text { "NON" à C } \\
\text { "OUI" à A }\end{array}$ & Echantillon & Ecart net \\
\hline Total nombre & 113 & 15 & 6 & 13 & 147 & 0,0476 \\
\hline Pourcentage & 76,87 & 10,20 & 4,08 & 8,84 & 100,00 & $\begin{array}{l}4,76 \\
1.61)\end{array}$ \\
\hline
\end{tabular}

Lecture: *: Significatif à 10\%; **: Significatif à 5\%. ***: Significatif à 1\%. Remarque : L'écart de $4.76 \%$ se trouve à la limite de significativité au seuil de $10 \%$ avec un t_student de 1.61 .

Notons que cet écart aurait dû être similaire avec le français ayant un Baccalauréat mais que l'effet est en fait inverse comme nous l'avons vu lors de l'analyse du couple AB.

\section{Hiérarchie dans l'ordre des réponses faites aux candidats}

Nous avons cherché à voir si une discrimination existe dans l'ordre des réponses faites aux candidats. Ainsi, nous avons traité de façon statistique et économétrique le sous échantillon de 41 entreprises qui ont répondu positivement aux candidats afin de voir s'il y des préférences dans l'ordre d'appel des candidats.

Parmi ces 41 entreprises, 14 ont répondu positivement aux 3 candidats, 15 ont répondu aux 2 candidats et 12 ont répondu à 1 seul candidat. 14 entreprises ont donc contacté les trois candidats. Parmi celles-ci, une seule entreprise a contacté le premier le candidat le plus formé avec une avance d'une semaine par rapport aux autres et une entreprise a contacté en premier le candidat français avec une avance d'un jour par rapport aux autres. Deux autres entreprises ont contacté le français d'origine maghrébine de niveau BAC après plus de 4 jours par rapport aux deux autres candidats. Ainsi, pendant au moins 4 jours, deux entreprises n'ont même pas pris en compte le candidat d'origine maghrébine avec des caractéristiques productives équivalentes au candidat d'origine français. 
Les 10 autres entreprises ont contacté les candidats le même jour, avec seulement un délai de quelques minutes ou quelques secondes. Ainsi, seulement 24\% des 41 employeurs ont choisi d'inviter tous les candidats aux entretiens d'embauche : 10 entreprises sur 41 ont répondu aux 3 candidats le même jour. Cependant, pour 9 recruteurs sur ces 10, le premier candidat contacté a été le français, soit 10 fois sur 14 (72\%).

Nous remarquons donc une forte disparité dans l'ordre dans lequel sont appelés les candidats. Le candidat le mieux qualifié n'est appelé que 3 fois en première position alors que le candidat français est appelé 10 fois en première position. Le candidat d'origine maghrébine n'ayant qu'un BAC n'est contacté en première position qu'une seule fois par mail quelques instants avant les deux autres candidats.

Afin de détailler cette tendance, nous avons décidé d'analyser le nombre de fois où le Français d'origine maghrébine avec BTS et le Français autochtone avec BAC sont appelés en premier lorsque les deux sont appelés. La tendance se confirme, le Français autochtone est appelé en premier position 15 fois sur 23 (14 entreprises qui ont appelé les 3 candidats plus 9 entreprises qui ont appelé seulement les candidats A et B) et le Français d'origine maghrébine seulement 7 fois. Enfin, nous avons analysé le nombre de fois où le Français d'origine maghrébine avec BAC et le Français autochtone avec BAC sont appelés en premier lorsque les deux sont appelés. La tendance est sans ambivalence, le Français autochtone est appelé en premier 14 fois sur 19 (14 entreprises qui ont appelé les 3 candidats plus 5 entreprises qui ont appelé seulement les candidats B et C) et le Français d'origine maghrébine seulement 2 fois.

En analysant la hiérarchie d'appels et combien de fois chaque candidat se trouve dans la première, deuxième ou troisième position à être appelé, la tendance est sans appel : le Français est majoritairement appelé en premier lorsque plusieurs candidats sont appelés (cf. tableau 9). 
Tableau 9

\section{Hiérarchie d'appels}

\begin{tabular}{|c|c|c|c|c|}
\hline \multicolumn{5}{|c|}{ Hiérarchie : cas où tous les 3 candidats sont appelés } \\
\hline Hiérarchie & $\mathbf{A}$ & B & $\mathbf{C}$ & $\begin{array}{l}\text { Total } \\
\text { entreprises }\end{array}$ \\
\hline 1er appelé & 3 & 10 & 1 & 14 \\
\hline 2ème appelé & 7 & 2 & 5 & 14 \\
\hline 3ème appelé & 4 & 2 & 8 & 14 \\
\hline \multicolumn{5}{|c|}{ Hiérarchie : cas d'au moins 2 candidats appelés } \\
\hline Hiérarchie & $\mathbf{A}$ & B & $\mathbf{C}$ & $\begin{array}{l}\text { Total } \\
\text { entreprises }\end{array}$ \\
\hline 1er appelé & 8 & 19 & 2 & 29 \\
\hline 2ème appelé & 12 & 7 & 10 & 29 \\
\hline 3ème appelé & 4 & 2 & 8 & 14 \\
\hline \multicolumn{5}{|c|}{ Hiérarchie : cas d'au moins 1 candidat appelé } \\
\hline Hiérarchie & $\mathbf{A}$ & B & $\mathbf{C}$ & $\begin{array}{l}\text { Total } \\
\text { entreprises }\end{array}$ \\
\hline 1er appelé & 12 & 26 & 3 & 41 \\
\hline 2ème appelé & 12 & 7 & 10 & 29 \\
\hline 3ème appelé & 4 & 2 & 8 & 14 \\
\hline
\end{tabular}

Le candidat d'origine française est visiblement le plus appelé en première position dans les trois cas. Sur l'ensemble des 41 entreprises il est appelé en première position 26 fois contre juste 3 fois pour un candidat d'origine maghrébine ayant les mêmes caractéristiques productives et 12 fois pour le candidat d'origine maghrébine ayant une formation supérieure aux autres.

\section{Étude de la discrimination dans l'ordre d'appel par couple des candidats}

Une autre approche est de mesurer la préférence pour l'un des trois candidats non pas juste par le nombre de fois à être appelé en première position mais par le nombre de fois à être préféré par couple de candidats. Nous avons donc cherché à voir quel candidat est appelé avant l'autre pour chaque couple de candidats (cf. tableau 10). 
Afin de réaliser cela, dans chaque couple, nous avons considéré comme « candidat préféré » :

- Le candidat appelé avant l'autre candidat du couple analysé.

- Le candidat du couple analysé qui est le seul à avoir été appelé.

- Le candidat du couple analysé qui est appelé avec le troisième candidat qui se trouve dehors du couple analysé.

Tableau 10

Ordre d'appel - candidat préféré

\begin{tabular}{|l|l|l|l|l|l|}
\hline Couple & Échantillon & $\mathbf{1}^{\text {er }} \mathbf{\text { vs 2 }} \mathbf{2}^{\mathbf{e}}$ & $\mathbf{2}^{\mathbf{e}} \mathbf{v s ~ \mathbf { 1 } ^ { \text { er } }}$ & Écart net \% & p value \\
\hline AB & 40 & 13 & 27 & $35,00^{* *}$ & 0,02 \\
\hline BC & 37 & 32 & 5 & $72,97^{* * *}$ & 0,00001 \\
\hline AC & 34 & 23 & 11 & $35,29^{* *}$ & 0,03 \\
\hline
\end{tabular}

Lecture : Test binomial exact de traitement égalitaire.

Seuils de significativité * : Significatif à 10\%; **: Significatif à 5\%; ***: Significatif à $1 \%$.

Sur 40 entreprises qui ont appelé au moins 1 des 2 candidats du couple AB, 13 ont appelé le candidat d'origine maghrébine avant le candidat d'origine française. 27 entreprises ont appelé le candidat d'origine française avant le candidat d'origine maghrébine. Nous concluons qu'il y a donc un écart net de $35 \%$ en faveur du candidat d'origine française avec une probabilité de commettre une erreur de 0.02 .

\section{Couple AB : effet origine + formation}

Le couple $\mathrm{AB}$ est formé des candidats suivants : A est le candidat d'origine maghrébine de niveau formation $\mathrm{BAC}+2$ et candidat $\mathrm{B}$ est le candidat d'origine français de niveau formation BAC.

40 entreprises ont appelé au moins 1 des 2 candidats A et B. L'échantillon est constitué ainsi :

- 14 entreprises ont appelé les 3 candidats A, B, C.

- 9 entreprises ont appelé les 2 candidats A et B.

- 7 entreprises ont appelé juste le candidat d'origine française (B avant A).

- 4 entreprises ont appelé juste le candidat d'origine maghrébine avec BTS (A avant B).

- 5 entreprises ont appelé les candidats B et C (B avant A).

- 1 entreprise a appelé les candidats A et C (A avant B).

Sur un échantillon de 40 entreprises qui ont contacté au moins l'un des deux candidats du couple AB, le candidat d'origine française est contacté 27 fois avant le candidat d'origine maghrébine en sachant que ce dernier est mieux formé. Celui-ci est appelé juste 13 fois avant le candidat d'origine française. 
Nous avons ainsi trouvé un écart significatif de 35\% entre les 2 candidats. Les employeurs préfèrent le candidat d'origine française de niveau formation $\mathrm{BAC}$ en défaveur du second candidat d'origine maghrébine, malgré le diplôme plus élevé qu'il détient (BTS). Un diplôme plus élevé ne « compense » pas l'origine maghrébine.

\section{Couple BC : effet origine}

Le couple BC est formé des candidats : B est le candidat d'origine française de niveau formation $\mathrm{BAC}$ et le candidat $\mathrm{C}$ est le candidat d'origine maghrébine du même niveau de formation BAC.

37 entreprises ont appelé au moins 1 des 2 candidats B et C. L'échantillon est constitué ainsi :

- 14 entreprises ont appelé tous les 3 candidats A, B, C.

- 5 entreprises ont appelé les candidats B et C.

- 7 entreprises ont appelé juste le candidat d'origine française (B avant C).

- 1 entreprise a appelé juste le candidat C (C avant B).

- 1 entreprise a appelé les candidats $\mathrm{A}$ et $\mathrm{C}(\mathrm{C}$ avant $\mathrm{B})$.

- 9 entreprises ont appelé les 2 candidats A et B (B avant C).

Sur un échantillon de 37 entreprises qui ont contacté au moins l'un des deux candidats du couple BC, le candidat d'origine française est contacté 32 fois avant le candidat d'origine maghrébine toutes choses étant égales par ailleurs. Le candidat d'origine maghrébine est appelé juste 5 fois avant le candidat d'origine française.

Nous avons ainsi trouvé un écart significatif de 75,97\% entre les 2 candidats. Il n'y a presque aucun doute que les employeurs préfèrent le candidat d'origine française en défaveur du second candidat d'origine maghrébine.

\section{Couple AC : effet formation}

Le couple AC est formé des candidats : A est le candidat d'origine maghrébine diplômé $\mathrm{BAC}+2$ et candidat $\mathrm{C}$ est le candidat d'origine maghrébine diplômé BAC.

34 entreprises ont appelé au moins 1 des 2 candidats A et C. L'échantillon est constitué ainsi :

- 14 entreprises ont appelé tous les 3 candidats A, B, C.

- 1 entreprise a appelé les candidats A et $\mathrm{C}$.

- 4 entreprises ont appelé juste le A (A avant C).

- 1 entreprise a appelé juste le candidat $\mathrm{C}$ (C avant $\mathrm{B})$.

- 9 entreprises ont appelé les 2 candidats A et B.

- 5 entreprises ont appelé les candidats B et C (C avant A). 
Parmi ces 34 entreprises qui ont contacté au moins l'un des deux candidats du couple AC, 23 ont appelé le candidat le mieux formé avant l'autre candidat. L'écart étant significatif, nous concluons que dans ce cas les employeurs préfèrent le candidat le mieux formé. Un diplôme plus élevé accorde donc plus de chances d'accéder à l'emploi.

\section{Une présomption de discrimination dans l'accès aux entretiens d'embauche...}

L'ensemble de ces résultats montre des effets propres à l'origine. Le premier constat est celui de l'ampleur des discriminations dont font l'objet les jeunes d'origine maghrébine. Selon leur origine, nous avons trouvé un écart important entre les candidats qui n'est que faiblement compensé par une qualification supérieure. Le candidat d'origine maghrébine de même niveau doit en moyenne envoyer presque deux fois plus de candidatures pour obtenir autant d'invitations à des entretiens d'embauche que le candidat dont les noms et prénoms évoquent l'origine française. Le candidat d'origine maghrébine mieux qualifié doit envoyer 1,25 fois plus de CV que le français alors qu'il aurait dû accéder à l'embauche plus facilement selon son niveau de qualification.

Le second constat est que les écarts sont différents selon la qualification des candidats d'origine maghrébine: le candidat le moins qualifié est beaucoup plus discriminé que le candidat plus qualifié. Cependant, le candidat maghrébin plus qualifié n'arrive pas à avoir les mêmes chances à l'embauche que le candidat français moins qualifié.

\section{...mais aussi dans l'ordre d'appel des candidats aux entretiens d'embauche}

Un autre élément nous a marqué pendant cette étude, lorsque plusieurs candidats sont appelés, c'est en grande majorité le Français qui est appelé en premier. On peut légitimement se demander si les candidats d'origine maghrébine auraient été appelés si le candidat français avait répondu positivement à l'invitation à un entretien. La discrimination n'est-elle pas plus forte que le présentent les simples taux de réussite ? Cette tendance n'est que très faiblement compensée par une qualification supérieure.

Sous la réserve qu'il est difficile de distinguer la discrimination statistique de la discrimination liée aux préférences, les écarts trouvés semblent suggérer la coexistence de ces deux types de discrimination. Ainsi, en comparant les deux candidats d'origine maghrébine, nous remarquons que lorsque l'on augmente la qualification, il y a plus de chances d'accéder à l'embauche car le candidat est plus productif. En revanche, en comparant le candidat 
d'origine maghrébine le plus diplômé avec le candidat d'origine française nous trouvons une préférence pour ce dernier, ce qui semble suggérer une discrimination liée aux préférences.

Enfin, une comparaison entre le candidat d'origine maghrébine et le candidat d'origine française ayant les mêmes caractéristiques productives montre une préférence pour le candidat d'origine française avec un écart plus grand que celui trouvé dans le deuxième cas. Cet écart pourrait être expliqué par une discrimination statistique due au manque d'information des employeurs sur la productivité des candidats. Ainsi, l'appréciation des compétences inobservables que le recruteur infère lors de la lecture d'une candidature repose sur ses croyances sur la productivité moyenne du groupe démographique auquel appartient le candidat.

Le candidat d'origine maghrébine le plus diplômé a un accès aux entretiens d'embauche inférieur au candidat d'origine française mais plus de chances que l'autre candidat d'origine maghrébine. Alors que le diplôme augmente les chances des candidats, l'origine maghrébine reste pourtant pénalisante.

Ces résultats suggèrent l'existence de la discrimination en raison de l'origine ethnique sur ce marché du travail, ils ne peuvent pas être généralisés sur l'ensemble du marché du travail. Ils indiquent l'ampleur de cette discrimination à l'embauche à un moment donné (fin juillet 2010 - fin août 2010) et dans le champ couvert par cette expérience (profession de technicien de maintenance) dans le contexte géographique de l'Île-de-France.

Le travail effectué pourrait bénéficier des perspectives de recherche suivantes. Au niveau du testing, il serait intéressant d'élargir cette étude à un nombre de candidatures plus large et à des métiers différents afin de confirmer les tendances observées sur notre échantillon. Il serait aussi souhaitable d'étudier l'impact de différents niveaux de qualification sur un emploi (Bac, BTS, Licence, Master, ...). Une autre piste intéressante serait d'étendre l'étude en ajoutant des données de position géographique (résidence dans une ZUS, résidence dans un quartier calme, ...) et d'analyser l'effet croisé de ces différentes variables. Enfin, concernant les résultats trouvés quant à l'ordre des réponses faites aux candidats, une étude intéressante serait de continuer cette analyse avec un modèle comme, par exemple, un modèle logit multinomial. 


\section{Bibliographie}

Arrow K. J. (1972), « The theory of job discrimination », in Ashenfelter O. A. \& Reeds A. (eds,)

Discrimination in Labor Markets, Princeton University Press, p. 3-33.

Aeberhardt R, D. Fougere, J. Pouget et R. Rathelot (2010), «L'emploi et les salaires des enfants d'immigrés ».

Economie et Statistique no. 433-434-2010, pages 31-46.

Aeberhardt R, J. Pouget (2011), « National Origin Differences in Wages and Hierarchical Positions: Evidence on French Full-Time Male Workers from a Matched Employer-Employee Dataset », Annales d'Economie et de Statistique, forthcoming. (Previous versions: IZA Discussion Paper No 2779 and CREST Working Paper n²007-3).

Ali M Ahmed, Andersson L., Hammarstedt M. : « Ethnic Discrimination in the Market Place of Small Business Transfers », Economics Bulletin.

Becker G. (1957), The Economics of Discrimination, University of Chicago Press.

Becker G (1964), Human Capital, A Theoretical and Empirical Analysis, with Special Reference to Education, NBER-Columbia University Press

Bertrand M. et Mullainathan S. (2004), « Are Emily and Greg More Employable than Lakisha and Jamal? A Field Experiment on Labor Market Discrimination », American Economic Review, vol. 94(4), p. 991-1013.

BIT (2007), « Les discriminations à raison de "l' origine" dans les embauches en France : une enquête nationale par tests de discrimination selon la méthode du Bureau international du travail », Miméo.

Cediey E., Foroni F., Garner H. (2008), « Discriminations à l'embauche fondées sur l'origine à l'encontre de jeunes français(es) peu qualifié(e)s. Une enquête nationale par tests de discrimination ou testing », Premières synthèses, $\mathrm{n}^{\circ}$ 06.3, Dares.

Centre d'Analyse Strategique (2007), Dossier «La discrimination saisie sur le vif : le testing », Horizons Stratégiques, n5, pages 6-91.

Beauchemin C., Hamel C., Lesne M., Simon P., et l'équipe de l'enquête TeO : « Les discriminations : une question de minorités visibles ».

Duguet, E., Y. l'Horty et P. Petit (2009), "L'apport du testing à la mesure des discriminations », Connaissance de l'Emploi, CEE, Août, (68).

Duguet, E., N. Leandri, Y. l'Horty et P. Petit (2007), « Discriminations à l'embauche : un testing sur les jeunes de banlieues d'Ile de France », Rapport pour le Centre d'Analyse Stratégique.

Duguet, E., L. du Parquet Y. l'Horty, P. Petit et F. Sari (2011), « Discriminations à l'embauche des jeunes franciliens et intersectionalité du sexe et de l'origine : les résultats d'un testing ».

Dayan J. L., Echardour A. et Glaude M. (1996), « Le parcours professionnel des immigrés en France : une analyse longitudinale », Économie et statistique, n 299. 
Fix M., Turner M. A. (1998), A national report card on discrimination in America: the role of testing, Washington, The Urban Institute.

Heran F., Sabeg Y. (2010), "Inégalités et discriminations - pour un usage critique et responsable de l'outil statistique", rapport du COMEDD (COmité pour la MEsure de la Diversité et des Discriminations)

Heckman J. (1998), "Detecting Discrimination”, Journal of Economic Perspectives, vol. 12, $\mathrm{n}^{\circ} 2$, pp. 101-116.

Kenney G. M., D. A. Wissoker (1994), « An Analysis of the Correlates of Discrimination Facing Young Hispanic Job-Seekers », American Economic Review, 84 (3), pp. 674-683.

Neumark D (2010) «Detecting Discrimination in Audit and Correspondence Studies » Department of Economics, UCI, NBER, and IZA working paper 16448

Marshall A. (1890), Principles of Economics

Petit P. (2003), « Comment évaluer la discrimination à l'embauche? », Revue Française d'Economie, 17 (3), pp. 55-87.

Phelps E. S. (1972), « The Statistical Theory of Racism and Sexism », American Economic Review, vol. 62, n 4, p. 659-661.

Richard J.-L. (2006), « Présomption de discrimination à l'encontre des enfants d'immigré(s) africain(s) », Migrations Société, vol. 18, n 105-106, mai - août.

Riach P. et Rich J. (1991), «Field Experiments of Discrimination in the Market Place », The Economics Journal, vol. 112, p. 480-518.

Riach P. et Rich J. (2002), « Testing for Racial Discrimination in the Labour Market », Cambridge Journal of Economics, vol. 15, p. 239-256.

Silberman R., Fournier I. (2006), « Jeunes issus de l'immigration : une pénalité à l'embauche qui perdure », Bref du Céreq, $\mathrm{n}^{\circ} 226$.

Smith R. (1977), Civic ideas: conflicting visions of citizenship inU.S. History, New Haven, Conn. : Yale University Press

Viprey M. (2002), « Les mécanismes de discrimination à l'égard des jeunes dont l'origine étrangère est réelle ou supposée », Revue de l'IRES, n 39, 2002/2. 


\section{Annexe 1}

Tableau 11

Estimation de la probabilité d'obtention d'une réponse positive - modélisation Logit

\begin{tabular}{|l|l|l|l|}
\hline Variables & Coefficients & Ecart-type & Pr > t \\
\hline Caractéristiques relatives aux entreprises et aux postes à pourvoir \\
\hline Appartenance à un groupe & -0.3959 & 0.6192 & 0.5227 \\
\hline Taille d'entreprise & -0.0475 & 0.0877 & 0.5882 \\
\hline Sexe du recruteur & -0.0317 & 0.0534 & 0.5530 \\
\hline Type de contrat (réf : Intérim) & \multicolumn{3}{|l}{} \\
\hline Contrat CDD & 0.2293 & 0.3471 & 0.5088 \\
\hline Contrat CDI & 0.1888 & 0.4171 & 0.6508 \\
\hline Caractéristiques relatives au CV & \multicolumn{3}{|l}{} \\
\hline Type candidature (CV et lettre de motivation) & -0.0111 & 0.7863 \\
\hline Code postal ville de candidats (réf. Bondy) & \multicolumn{3}{|l}{} \\
\hline Le Blanc Mesnil & -0.0505 & 0.0409 & 0.3845 \\
\hline Noisy-le-Sec & 0.0505 & 0.0580 & 0.3845 \\
\hline Nombre d'observations & 441 & \\
\hline Nombre de groupes & 147 & \\
\hline
\end{tabular}

Lecture : Estimations par l'analyse du maximum de vraisemblance, model Logit.

Seuils de significativité * : Significatif à 10\% ; **: Significatif à 5\%; *** : Significatif à $1 \%$.

Les variables pouvant potentiellement expliquer une discrimination conditionnelle (variables relatives au poste à pourvoir, aux entreprises et à la candidature) apparaissent comme non significatives. 


\section{Annexe 2}

Nous présentons ci-après un CV et une lettre de motivation d'un des trois candidats fictifs qui ont candidaté sur des postes de technicien de maintenance.

\section{Nessim BELAHDEB}

19, Rue André Bernard

93156 Le Blanc Mesnil

Contact : Xx.XX.Xx.xx.Xx

Mail : xxx@ hotmail.fr

\section{Lettre de motivation}

\section{Objet : Candidature à l'emploi}

Madame, Monsieur,

Étant électrotechnicien de formation et ayant occupé plusieurs postes dans le domaine de la maintenance, je souhaiterais vous proposer ma candidature en réponse à votre annonce.

J'ai passé le BEP métiers de l'électrotechnique ainsi que le Bac Professionnel électrotechnique, énergie, équipements communicants. J'ai appris mon métier de technicien de maintenance en travaillant dans différentes entreprises.

Je suis sérieux et compétent et je souhaite continuer mon métier au sein de votre entreprise. Je m'adapte rapidement au travail autonome ou en équipe.

Dans l'attente d'une réponse de votre part veuillez agréer, Madame, Monsieur, l'expression de mon profond respect.

Nessim BELAHDEB 


\section{Nessim BELAHDEB}

19, Rue André Bernard

93156 Le Blanc Mesnil

Contact : 0645216490

Mail : n.belahdeb@hotmail.fr

\section{Technicien de Matntenance}

\section{INFORMATIONS}

\section{GENERALES}

Date de naissance :

Nationalité :

Situation de famille :

Permis :

Rayon de mobilité :
Né en 1981

Française

Célibataire sans enfant

Permis B

Ile-de-France

\section{Formation}

1998 - 2000 :

1996 - 1998 :

Lycée professionnel Le Foyer de Cachan

Bac Professionnel électrotechnique, énergie, équipements communicants

Lycée professionnel Francoise Cabrini, Noisy-le-Grand

BEP métiers de l'électrotechnique

\section{EXPERIENCES}

Depuis Oct. 2006 :

Janv. 2004 - Oct. 2006 :

Sept. 2000 - Déc. 2003 :

Sept. 1998 - Juillet 2000 :
Technicien de maintenance, Pro Maintenance, Champigny-sur-Marne

- Implantation et utilisation des équipements électriques

- Maintenance des installations et équipements

- Tests, vérifications et réglages des installations et équipements

\section{Technicien de maintenance, Installo Serv, Aulnay-sous-Bois}

- Maintenance et entretien générale des diverses installations

- Vérification et réglage des matériels et dispositifs

- Travaux de bricolage

$\underline{\text { Technicien de maintenance, Songera Tec, Vitry-sur-Seine }}$

- Mise en place et maintenance des dispositifs électriques

- Raccordement des matériels

- Préparation des devis et des rapports techniques

$\underline{\text { Technicien de maintenance (alternance) - Techno Savelys, Arcueil }}$

- Raccordements et câblages

- Utilisation et implantation des équipements

\section{Competences}

\begin{tabular}{ll}
\hline Compétences & - Tableur et traitement du texte \\
informatiques : & - Internet, Office, Windows
\end{tabular}

Autres compétences :

Compétences

- Bon bricoleur 
12-1. Etre meilleur Apprenti de France : Quels effets sur l'accès à l'emploi ? Les enseignements de deux expériences contrôlées sur des jeunes d'Ile-de-France Pascale Petit, Florent Fremigacci, Loïc du Parquet, Guillaume Pierne

12-2. L'intermédiation financière dans l'analyse macroéconomique : Le défi de la crise Eleni Iliopoulos, Thepthida Sopraseuth

12-3. Evaluer un dispositif sectoriel d'aide à l'emploi : L'exemple des hôtels cafés restaurants de 2004 à 2009

Mathieu Bunel

12-4. Evaluer les réformes des exonérations générales de cotisations sociales Mathieu Bunel, Céline Emond, Yannick L'Horty 
11-1. Les effets du lieu de résidence sur l'accès à l'emploi: Une expérience contrôlée sur des jeunes qualifiés en Ile-de-France

Yannick L'Horty, Emmanuel Duguet, Loïc du Parquet, Pascale Petit, Florent Sari

11-2. Comment développer les emplois favorables à la biodiversité en Ile-De-France ? Jean de Beir, Céline Emond, Yannick L'Horty, Laëtitia Tuffery

11-3. Être mobile pour trouver un emploi ?Les enseignements d'une expérimentation en région parisienne

Loïc du Parquet, Emmanuel Duguet, Yannick L’Horty, Pascale Petit, Florent Sari

11-4. Ce que font les villes pour les ménages pauvres. Résultats d'une enquête nationale sur les communes de plus de 20000 habitants

Denis Anne, Céline Emond, Yannick L’Horty

11-5. Discriminations à l'embauche des jeunes franciliens et intersectionalité du sexe et de l'origine: Les résultats d'un testing

Pascale PETIT, Emmanuel DUGUET, Yannick L'HORTY, Loïc du PARQUET, Florent

SARI

11-6. Les effets du bénévolat sur l'accès à l'emploi. Une expérience contrôlée sur des jeunes qualifiés d'Ile-de-France

Jonathan Bougard, Thomas Brodaty, Céline Emond, Yannick L’Horty, Loïc du Parquet et

Pascale Petit

11-7. « 10000 permis pour réussir ». Evaluation quantitative

Yannick L’Horty, Emmanuel Duguet, Sophie Kaltenmark, Pascale Petit

11-8. Why is there a faster return to work near the border?

Jonathan Bougard

11-9. Evaluer l'impact d'un micro-programme social : une étude de cas expérimentale Yannick L'Horty, Emmanuel Duguet, Pascale Petit

11-10. Les effets des aides publiques aux Hôtels Cafés Restaurants et leurs interactions : Une évaluation sur micro-données d'entreprises

Mathieu Bunel, Yannick L'Horty

11-11. Pourquoi tant de chômeurs à Paris

Yannick L'Horty, Florent Sari

11-12. LE WIKI IO : Réduire les risques de décrochage et d'abandon à la sortie du collège

Solène Coursaget, Emmanuel Duguet, Yannick L'Horty, Pascale Petit, Emmanuel Quenson

11-13. Le grand Paris de l'emploi

Yannick L'Horty, Florent Sari

11-14. Quelle politique publique pour protéger la biodiversité Jean De Beir, Céline Emond, Yannick L'Horty, Laetitia Tuffery 
La Fédération de recherche CNRS Travail, Emploi et Politiques Publiques (TEPP, FR $\mathbf{n}^{\circ} \mathbf{3 1 2 6}$ ) réunit des centres de recherche en économie et sociologie :

- Le Centre d'Etudes des Politiques Economiques de l'université d'Evry, EPEE, Université d'Evry Val d'Essonne

- Le Centre Pierre Naville, CPN, Université d'Evry Val d'Essonne

- Le Centre de Recherche en Economie et Management, CREM, Université de Caen Basse Normandie et Université de Rennes 1

- L'Equipe de Recherche sur les Marchés, l'Emploi et la Simulation, ERMES, Université deParis II Panthéon-Assas

- L'Equipe de Recherche sur l'Utilisation des Données Temporelles en Economie, ERUDITE, Université de Paris-Est Créteil et Université de Paris-Est Marne-la-Vallée

- Le Groupe d'Analyse des Itinéraires et des Niveaux Salariaux, GAINS, Université du Maine

La Fédération TEPP rassemble 150 chercheurs et enseignants-chercheurs, 140 doctorants et 40 chercheurs associés, qui étudient les mutations du travail et de l'emploi en relation avec les choix des entreprises et analysent les politiques publiques en mobilisant les nouvelles méthodes d'évaluation. 